\title{
Measurement of the specific heat and determination of the thermodynamic functions of relaxed amorphous silicon
}

\author{
P. Roura, ${ }^{1, a)}$ F. Taïr, ${ }^{2}$ J. Farjas, ${ }^{1}$ and P. Roca i Cabarrocas ${ }^{3}$ \\ ${ }^{1}$ GRMT, Department of Physics, University of Girona, Montilivi Campus, E17071 Girona, Catalonia, Spain \\ ${ }^{2}$ Département de physique, Université des Sciences et de la Technologie d'Oran USTO Oran, Algerie \\ ${ }^{3}$ LPICM, Ecole Polytechnique, 91128 Palaiseau, France
}

(Received 20 March 2013; accepted 19 April 2013; published online 7 May 2013)

\begin{abstract}
The specific heat, $c_{p}$, of two amorphous silicon (a-Si) samples has been measured by differential scanning calorimetry in the $100-900 \mathrm{~K}$ temperature range. When the hydrogen content is reduced by thermal annealing, $c_{p}$ approaches the value of crystalline $\mathrm{Si}$ (c-Si). Within experimental accuracy, we conclude that $c_{p}$ of relaxed pure a-Si coincides with that of $\mathrm{c}-\mathrm{Si}$. This result is used to determine the enthalpy, entropy, and Gibbs free energy of defect-free relaxed a-Si. Finally, the contribution of structural defects on these quantities is calculated and the melting point of several states of a-Si is predicted. (C) 2013 AIP Publishing LLC. [http://dx.doi.org/10.1063/1.4803888]
\end{abstract}

\section{INTRODUCTION}

Amorphous silicon (a-Si) and its hydrogenated version (a-Si:H) hold a high scientific interest because of their use in many applications such as solar cells and thin film transistors for large area displays, and because they are taken as a model to understand the structure of tetrahedral amorphous materials.

At present, the theoretical models of a-Si can be tested against a large amount of experimental data obtained from structural techniques, such as neutron and x-ray diffraction, Raman spectroscopy or electron paramagnetic resonance, which give key details about short-range and medium-range order or structural defects. ${ }^{1}$ In contrast, knowledge of its thermodynamic properties is very poor.

This situation may be due to the difficulty of doing thermal measurements on thin films and because a-Si is intrinsically unstable under heating as it tends to relax to microscopic configurations of lower energy and, ultimately, to crystallize. ${ }^{2}$ Consequently, thermodynamic data are scarce and not reliable enough to serve as solid tests for the microscopic models or to be used in practical applications where a-Si coexists in thermal equilibrium with other phases like in a-Si nanoparticles embedded in $\mathrm{SiO}_{2}{ }^{3}$

Determination of a-Si thermodynamic functions needs its entropy and enthalpy at a given temperature and its specific heat to be determined. The enthalpy at the crystallization temperature has recently been shown to vary between 5.6 and $13.5 \mathrm{~kJ} / \mathrm{mol}$ for relaxed a-Si, depending on the particular a-Si material ${ }^{4}$ with the minimum value being below $1 / 2$ the value usually taken as standard. ${ }^{5}$ All calculations made so far ${ }^{6,7}$ rely on this "standard" value, on a theoretical value of the entropy at $0 \mathrm{~K}$ (Ref. 8) and on specific heat values estimated from those of amorphous Ge. ${ }^{9}$ The specific heat of a-Si has only been measured at cryogenic temperatures and significant discrepancies arise between the several published values. ${ }^{10-12}$ Measurements above room temperature are more difficult to

\footnotetext{
a) Author to whom correspondence should be addressed. Electronic mail: pere.roura@udg.cat
}

do because, in fact, one is interested in the small excess specific heat with respect to crystalline silicon (c-Si).

In this paper, we will report on the experimental determination of the excess specific heat of a-Si in the 100-900 K temperature range. After a brief description of the experimental methods, our results will be given and the discrepancy with those values already published in the literature will be discussed. Our values will be then used to determine the excess Gibbs free energy of a-Si relative to $\mathrm{c}-\mathrm{Si}$ and to predict its melting temperature. Finally, some concluding remarks will follow.

\section{EXPERIMENTAL METHODS}

Two samples of a-Si:H were deposited on the inner walls of a plasma enhanced chemical vapor deposition (PECVD) reactor chamber and were collected in the form of flakes. X-ray diffraction (Bruker AXS Smart Apex diffractometer) revealed that they were $100 \%$ amorphous. Their hydrogen content, $\mathrm{n}_{\mathrm{H}} / \mathrm{n}_{\mathrm{Si}}$, was determined by elementary analysis (EA2400 Perkin Elmer). Hydrogen desorption during heating ramps was monitored by evolved gas analysis (EGA) experiments carried out in vacuum. Hydrogen was detected with an MKS quadrupole mass spectrometer (Microvision Plus).

The excess specific heat of a-Si with respect to c-Si, $\Delta c_{p} \equiv c_{p}(a-S i)-c_{p}(c-S i)$, was measured by differential scanning calorimetry (DSC) with the procedure described elsewhere $^{13}$ that allows detection of $\Delta c_{p}$ values as low as $1 \%$. For these experiments, large amounts of sample $(20-60 \mathrm{mg})$ were kept in platinum crucibles. Independent tests have been carried out to calibrate the apparatus sensitivity at these conditions. Two DSC apparatus were used: Mettler Toledo DSC821 and TA Instruments Q2000. The last DSC allowed us to do experiments at cryogenic temperatures.

\section{RESULTS: THE EXCESS SPECIFIC HEAT OF a-Si}

We studied two samples. Since they differ on their crystallization enthalpy $[9.6 \mathrm{~kJ} / \mathrm{mol}$ (sample A) and $7.3 \mathrm{~kJ} / \mathrm{mol}$ (sample B)], they give us the opportunity to analyze if this property has an effect on $\Delta c_{p}$ or not. 
Hydrogen atoms introduce more vibrational modes in the material. Consequently, it is natural to observe a diminution of $\Delta c_{p}$ when hydrogen content is reduced. We measured $\Delta c_{p}$ of sample A as a function of hydrogen content. To this aim, the sample was previously annealed at $10 \mathrm{~K} / \mathrm{min}$ up to 870 and $920 \mathrm{~K}$. We verified that, according to previous results, ${ }^{14,15}$ after annealing at $920 \mathrm{~K}$ the crystalline fraction was negligible. Fig. 1 makes clear that, as expected, annealing reduces $\Delta c_{p}$ considerably: after annealing at $920 \mathrm{~K}, \Delta c_{p}$ is reduced to $1 / 5$ of its as-grown value. Since elemental analysis and the EGA curve of Fig. 2 allows the hydrogen content to be determined, the dependence of $\Delta c_{p}$ on $\mathrm{n}_{\mathrm{H}} / \mathrm{n}_{\mathrm{Si}}$ can be plotted (inset of Fig. 1). From this plot, one can deduce that a large fraction of $\Delta c_{p}$ of the as-grown sample is due to the hydrogen atoms. Furthermore, extrapolation to $n_{H} / n_{S i}=0$ seems to indicate that $\Delta c_{p}$ for pure a-Si is near zero. This prediction has been verified experimentally; in a similar experiment, $\mathrm{n}_{\mathrm{H}} / \mathrm{n}_{\mathrm{Si}}$ has been further reduced by heating sample $A$ up to $920 \mathrm{~K}$ six times (now, $\mathrm{n}_{\mathrm{H}} / \mathrm{n}_{\mathrm{Si}}$ is under the sensitivity limit of the experiment, $0.2 \%$ ). The result is that, in this condition, $\Delta c_{p}$ becomes zero above $570 \mathrm{~K}$ and has a very small value $\left[<0.2 \mathrm{~J} / \mathrm{K} / \mathrm{mol}\right.$, i.e., $\left.<1 \% \mathrm{c}_{\mathrm{p}}(\mathrm{c}-\mathrm{Si})\right]$ below this temperature (Fig. 1).

Finally, sample B delivered similar results. Here again, we verified that $\Delta c_{p}$ diminished when $\mathrm{n}_{\mathrm{H}}$ was reduced by thermal annealing and its value became negligible after complete dehydrogenation. In this state, $\Delta c_{p}$ of sample B was measured within a wider temperature range (120-770 K). The results that are shown as star symbols in Fig. 3 agree with those of dehydrogenated sample A (Fig. 1). To better appreciate the significance of our results, we should say that previous estimations of $\Delta c_{p}$ based on measurements on a-Ge gave values of $1.4 \mathrm{~J} / \mathrm{K} / \mathrm{mol}$ at $570 \mathrm{~K}^{6}$

\section{ANALYSIS: THERMODYNAMIC FUNCTIONS AND MELTING TEMPERATURE OF RELAXED a-Si}

In this section, we want to determine the thermodynamic functions of "defect-free relaxed a-Si" (henceforth labeled

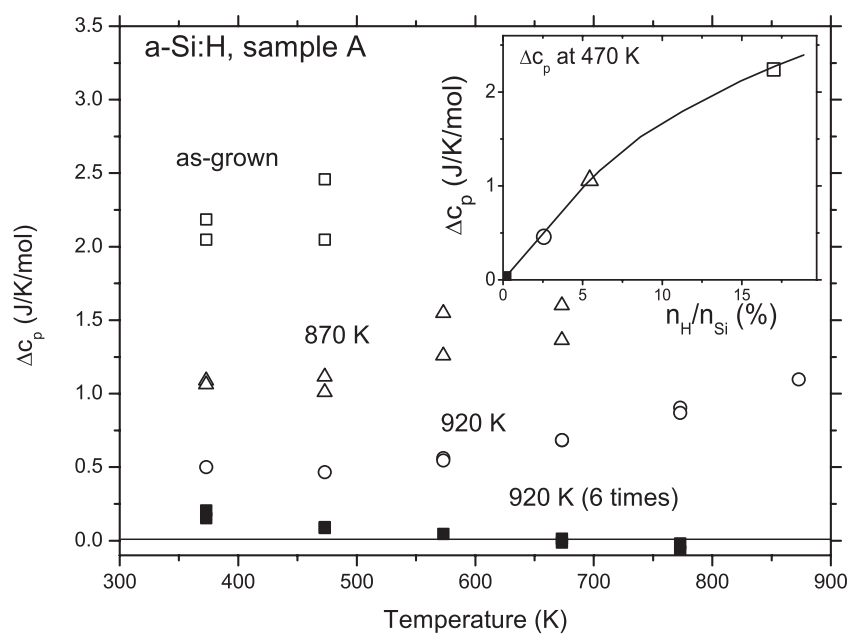

FIG. 1. Excess specific heat of a-Si:H, $\Delta c_{p}$, as a function of the temperature measured after annealing sample $\mathrm{A}$ at different temperatures to reduce the hydrogen content. After annealing it 6 times at $920 \mathrm{~K}, \mathrm{n}_{\mathrm{H}}$ became negligible. Inset: dependence of $\Delta c_{p}$ on the hydrogen content.

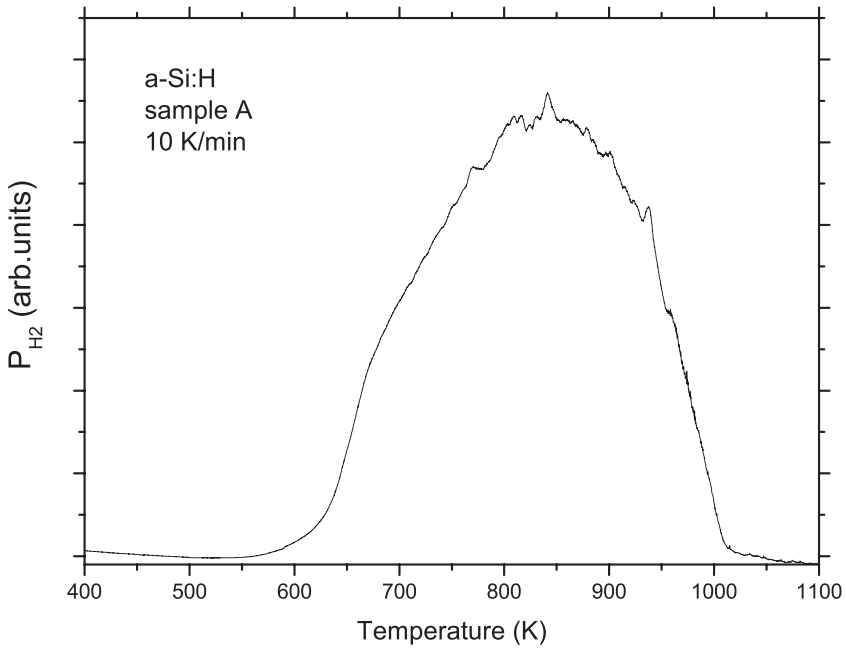

FIG. 2. Evolved gas analysis curve of dehydrogenation for sample A.

with a superscript "df"). This state corresponds to a microscopic configuration with minimum bond-angle strain (the so-called "relaxed state") and with negligible density of structural defects; it can be viewed as the microscopic configuration closest to c-Si. We will show that our experimental values of $\Delta c_{p}$, together with published values of the crystallization enthalpy ${ }^{4}$ and the entropy of a-Si, ${ }^{8}$ make it possible to propose the most reliable values of its thermodynamic functions, published so far. In a second step, we will estimate the contribution of structural defects and, finally, the melting temperature of relaxed a-Si will be predicted. It is worth noting that melting is the only reversible transformation of a-Si that has been detected so far $^{16}$ and that it could be used to test our proposed thermodynamic functions.

The enthalpy of a-Si relative to c-Si is given by

$$
\Delta H_{a c}(T)=\Delta H_{a c}\left(T_{0}\right)+\int_{T_{0}}^{T} \Delta c_{p} d T^{\prime},
$$

where $\Delta H_{a c}\left(T_{0}\right)$ can be taken as the heat released during crystallization at $\mathrm{T}_{0}$. The entropy difference with c-Si can

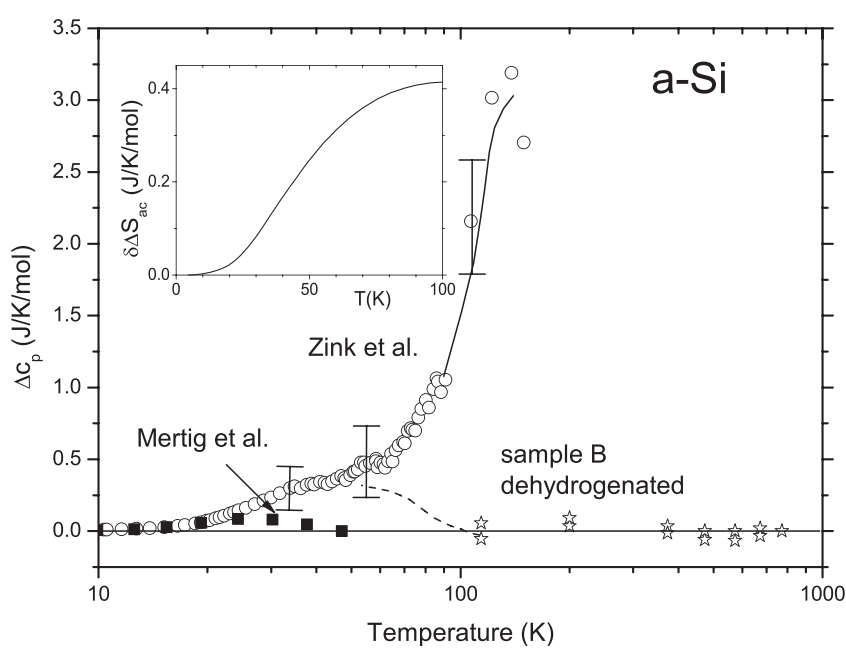

FIG. 3. Excess specific heat of dehydrogenated sample B for $\mathrm{T}>120 \mathrm{~K}$ (stars). The open points and full squares are taken from the literature. Inset: upper bound for the entropy variation up to $100 \mathrm{~K}$. 
also be evaluated if we know $\Delta c_{p}$ and the difference at a single temperature,

$$
\Delta S_{a c}(T)=\Delta S_{a c}\left(T_{0}\right)+\int_{T_{0}}^{T} \frac{\Delta c_{p}}{T^{\prime}} d T^{\prime} .
$$

And, finally, the Gibbs free energy can be calculated from its definition,

$$
\Delta G_{a c}(T)=\Delta H_{a c}(T)-T \Delta S_{a c}(T) .
$$

The first difficulty one encounters when trying to determine the thermodynamic functions of a-Si is that they are not unique. It is well known that the energy of a-Si obtained by ion implantation diminishes steadily upon thermal annealing due to structural relaxation. ${ }^{2}$ Even for well relaxed material (i.e., material with minimum bond $\operatorname{strain}^{17}$ ), the crystallization enthalpy varies within a factor of two due to the varying densities of structural defects. ${ }^{4}$ So, the minimum value of the crystallization enthalpy is $5.6 \mathrm{~kJ} / \mathrm{mol}$ at $\mathrm{T}_{0}=1000 \mathrm{~K}$ and corresponds to $\Delta H_{a c}^{d f}\left(T_{0}\right)$. The excess enthalpy above this value is the contribution of structural defects. ${ }^{18}$ It can be as high as $7 \mathrm{~kJ} / \mathrm{mol}$. Although the nature and formation energy of these structural defects has not been elucidated, we know that they are not paramagnetic and, consequently, they cannot contain an odd number of dangling bonds. Single vacancies have formation energy around $4 \mathrm{eV}$ (Ref. 19), they should have a density of $n_{d}=1.7 \%$ to explain the excess crystallization enthalpy. This value agrees with the density of defects that remain in a-Si obtained by ion implantation after thermal annealing ${ }^{20}$ and will be taken as representative of the contribution of defects to the a-Si thermodynamic functions.

The second difficulty is the entropy value of a-Si at a single temperature, $\Delta S_{a c}\left(T_{0}\right)$. A theoretical estimate obtained from defect-free microscopic models of a-Si is $1.7 \mathrm{~J} / \mathrm{K} / \mathrm{mol}^{8}{ }^{8}$ No other value has been proposed in the literature neither from experimental nor from theoretical grounds. So, we take it as being $\Delta S_{a c}^{d f}(0 \mathrm{~K})$. The entropy increases above this value due to structural defects according to

$$
\Delta S_{d}=-R n_{d} \ln \left(n_{d}\right),
$$

where $\mathrm{R}$ is the gas constant $(8.31 \mathrm{~J} / \mathrm{K} / \mathrm{mol})$ and subindex $d$ refers to "defects." For $\mathrm{n}_{\mathrm{d}}=1.7 \%, \Delta S_{d}=0.58 \mathrm{~J} / \mathrm{K} / \mathrm{mol}$.

The third difficulty comes from the uncertainty in the value of $\Delta c_{p}$. In Fig. 3 we have plotted, in addition to our results, the values measured by Zink et al. ${ }^{11}$ on an evaporated film and those of Mertig et al. ${ }^{12}$ In contrast to our present results, the film of Zink has a positive excess specific heat. Although the origin of this discrepancy cannot be known, we should remark that the film was not annealed and, consequently, it could be far from the relaxed state. Since we have not measured $\Delta c_{p}$ below $100 \mathrm{~K}$, we can take the values of Zink et al. as reference for an upper bound to $\Delta c_{p}$ for relaxed a-Si. We consider that the dashed line in Fig. 3 is a reasonable upper bound. Furthermore, the two samples we measured are not defect-free. Although they have enthalpies that exceed $\Delta H_{a c}^{d f}\left(T_{0}\right)$ by 4 and $1.7 \mathrm{~kJ} / \mathrm{mol}$ (samples A and $\mathrm{B}$, respectively), their $\Delta c_{p}$ values are very close to zero.
So, we will consider that this upper bound is valid for defectfree a-Si as well as for a-Si containing structural defects.

Measurement of $\Delta c_{p}$ allows determination of the temperature variation of $\Delta H_{a c}$ and $\Delta S_{a c}$ through the application of Eqs. (1) and (2). According to the upper bound established above, variation of these magnitudes can only take place below $100 \mathrm{~K}$. In the case of $\Delta H_{a c}$, Eq. (1) delivers a negligible maximum variation of $0.02 \mathrm{~kJ} / \mathrm{mol}$, whereas the variation of $\Delta S_{a c}$ between 0 and $100 \mathrm{~K}, \delta \Delta S_{a c}$, is more substantial but below $0.4 \mathrm{~J} / \mathrm{K} / \mathrm{mol}$ (see inset of Fig. 3).

The $\Delta S_{a c}$ and $\Delta H_{a c}$ values calculated so far will serve to determine the Gibbs free energy of relaxed a-Si (Eq. (3)). The results have been plotted in Fig. 4 together with the free energy of liquid $\mathrm{Si}, \Delta G_{l c}$. The intersection of the $\Delta G_{l c}$ and $\Delta G_{a c}$ curves delivers the melting point, $\mathrm{T}_{\mathrm{al}}$, of the various states of relaxed a-Si. Defect-free a-Si melts at a temperature quite close to $c-S i, T_{c l}-T_{a l}=70-95 \mathrm{~K}$. On the other hand, structural defects have a very pronounced effect on the melting temperature that can be $350 \mathrm{~K}$ below $\mathrm{T}_{\mathrm{cl}}$.

The only experimental determination of the melting point of a-Si $\left[\mathrm{T}_{\mathrm{al}}(\mathrm{exp})-\mathrm{T}_{\mathrm{cl}}=200 \pm 50 \mathrm{~K}\right]$ was carried out on Si amorphized by ion implantation, ${ }^{16}$ and both experiment ${ }^{21}$ and theory ${ }^{22}$ indicate that the film relaxed before melting. So, this value can be compared with our predictions in Fig. 4. This comparison indicates that, although this film was relaxed, it contained a density of defects high enough to have a significant effect on the melting point.

Finally, we must say that in the literature the melting point of a-Si has been only analyzed in terms of structural relaxation. Experiments have shown that unrelaxed a-Si melts at $115 \mathrm{~K}$ below relaxed a-Si. ${ }^{21}$ The present work shows that the density of defects is an additional structural parameter that has to be taken into account. By taking it into account, we have predicted that $T_{a l}$ spans over a range of

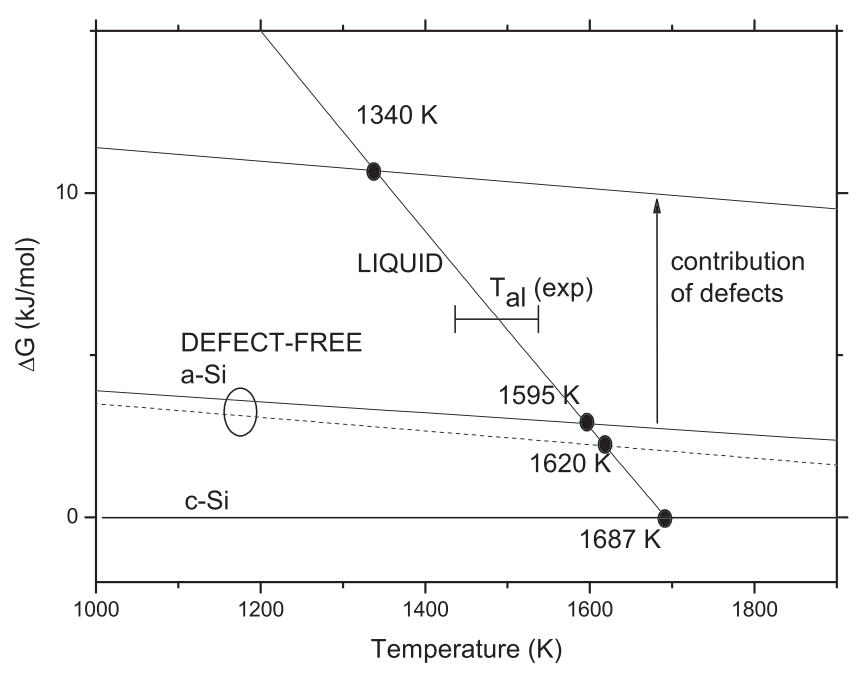

FIG. 4. Excess Gibbs free energy of relaxed a-Si. The dotted line takes into account the maximum contribution of $\Delta c_{p}$. The upper curve is the free energy for the a-Si with maximum crystallization enthalpy (maximum density of structural defects) reported so far. ${ }^{4}$ Intersection with the curve of liquid Si delivers the expected melting points of a-Si that can be compared to the melting point of c-Si $(1687 \mathrm{~K})$. The horizontal bar is the only experimental determination of $\mathrm{T}_{\mathrm{al}}$. 
$280 \mathrm{~K}$, meaning that the density of defects has a stronger influence on $\mathrm{T}_{\mathrm{al}}$ than the degree of relaxation.

\section{CONCLUSIONS}

The measurements of the excess specific heat of a-Si with respect to c-Si, $\Delta c_{p}$, reported here, led us to conclude that above $100 \mathrm{~K}, \Delta c_{p}$ becomes negligible when samples have been previously dehydrogenated (and relaxed). This conclusion does not depend on the density of structural defects (i.e., it is independent of the sample crystallization enthalpy). Below $100 \mathrm{~K}$, an upper bound to $\Delta c_{p}$ has been established that limits the maximum variation of the enthalpy and entropy with temperature. These results, together with previous measurements of the crystallization enthalpy, ${ }^{4}$ make clear that, from the thermodynamic point of view, relaxed a-Si is much closer to $\mathrm{c}-\mathrm{Si}$ than was previously thought. Finally, the melting point of a-Si has been predicted and found to be very close to that of c-Si for defect-free relaxed material. Furthermore, it is concluded that the density of defects has a more pronounced effect on the melting temperature than the degree of relaxation does.

\section{ACKNOWLEDGMENTS}

This work was partially funded by the Generalitat de Catalunya (Contract No. 2009SGR-185). The authors are indebted to Dr. Joan Pere López Olmedo of STR, University of Girona, for his technical assistance.

${ }^{1}$ R. Singh, S. Prakash, N. N. Shukla, and R. Prasad, Phys. Rev. B 70, 115213 (2004).
${ }^{2}$ S. Roorda, S. Doorn, W. C. Sinke, P. M. L. O. Scholte, and E. van Loenen, Phys. Rev. Lett. 62, 1880 (1989).

${ }^{3}$ A. Sarikov and M. Zacharias, J. Phys.: Condens. Matter 24, 385403 (2012).

${ }^{4}$ F. Kail, J. Farjas, P. Roura, C. Secouard, O. Nos, J. Bertomeu, and P. Roca i Cabarrocas, Phys. Status Solidi (RRL) 5, 361 (2011).

${ }^{5}$ E. P. Donovan, F. Spaepen, J. M. Poate, and D. C. Jacobson, Appl. Phys. Lett. 55, 1516 (1989).

${ }^{6}$ E. P. Donovan, F. Spaepen, D. Turnbull, J. M. Poate, and D. C. Jacobson, J. Appl. Phys. 57, 1795 (1985).

${ }^{7}$ D. Choudhary and P. Clancy, J. Chem. Phys. 122, 174509 (2005).

${ }^{8}$ F. Spaepen, Philos. Mag. 30, 417 (1974).

${ }^{9}$ H. S. Chen and D. Turnbull, J. Appl. Phys. 40, 4214 (1969).

${ }^{10}$ J. E. Graebner, B. Golding, L. C. Allen, J. C. Knights, and D. K. Biegelsen, Phys. Rev. B 29, 3744 (1984).

${ }^{11}$ B. L. Zink, R. Pietri, and F. Hellman, Phys. Rev. Lett. 96, 055902 (2006).

${ }^{12}$ M. Mertig, G. Pompe, and E. Hegenbarth, Solid State Commun. 49, 369 (1984).

${ }^{13}$ P. Roura, D. Sanchez-Rodriguez, and J. Farjas, Thermochim. Acta 522, 161 (2011).

${ }^{14}$ J. Farjas, N. Butchosa, and P. Roura, J. Therm. Anal. Calorim. 102, 615 (2010).

${ }^{15}$ F. Kail, J. Molera, J. Farjas, P. Roura, C. Secouard, and P. Roca i Cabarrocas, J. Phys.: Condens. Matter 24, 095401 (2012).

${ }^{16}$ M. O. Thompson, G. J. Galvin, J. W. Mayer, P. S. Peercy, J. M. Poate, D. C. Jacobson, A. G. Cullis, and N. G. Chew, Phys. Rev. Lett. 52, 2360 (1984).

${ }^{17}$ F. Kail, J. Farjas, P. Roura, C. Secouard, O. Nos, J. Bertomeu, F. Alzina, and P. Roca i Cabarrocas, Appl. Phys. Lett. 97, 031918 (2010).

${ }^{18}$ P. Roura, J. Farjas, and P. Roca i Cabarrocas, J. Appl. Phys. 104, 073521 (2008).

${ }^{19}$ P. Roura, J. Farjas, Ch. Rath, J. Serra-Miralles, E. Bertran, and P. Roca i Cabarrocas, Phys. Rev. B 73, 085203 (2006).

${ }^{20}$ P. A. Stolk, F. W. Saris, A. J. M. Berntsen, W. F. van der Weg, L. T. Sealy, R. C. Barklie, G. Krötz, and G. Muller, J. Appl. Phys. 75, 7266 (1994).

${ }^{21}$ M. G. Grimaldi, P. Baeri, and M. A. Malvezzi, Phys. Rev. B 44, 1546 (1991).

${ }^{22}$ P. Roura and J. Farjas, Acta Mater. 57, 2098 (2009). 